\title{
Insulintherapie bei Typ-2-Diabetes - so spät wie möglich!
}

\author{
Stephan Jacob
}

Betazell-Verlust - eine veraltete These I Eine HOMA- $\beta$-Analyse der UK Prospective Diabetes Study (UKPDS) zeigte mit der Dauer des Typ-2 Diabetes einen zunehmenden Betazell-Verlust [1]. Aus dieser Beobachtung wurde fast ein Dogma, das sich über viele Jahre gehalten hat:

- Je länger der Diabetes besteht, umso eher komme es zum Betazell-Versagen.

- Daher solle/müsse sinnvollerweise eine Insulin-Substitution erfolgen.

Auch wurde angenommen, dass sich eine frühe Insulin-Therapie günstig auf die (Rest-) BetazellFunktion und die Stoffwechselkontrolle auswirkt.

Reversible Betazell-Dysfunktion I In den letzten Jahren haben sich die Erkenntnisse der Forschung tiefgreifend gewandelt. Die Verfügbarkeit neuer Therapieansätze verändert möglicherweise auch den bisher als „natürlich“ angesehenen Krankheitsverlauf. Neuere Endpunkt-Studien [2] haben zudem weitere Fragen und Zweifel am Vorteil einer frühen Insulintherapie aufgeworfen. Immer mehr Studien stellen das alte Weltbild des (permanenten) Verlustes der Insulinsekretion in Frage und diskutieren eher eine Betazell-Dysfunktion als einen Betazell-Verlust. So hat eine Änderung der Mahlzeitenfolge (z.B. großes vs. kleines Abendessen) u.a. die Insulin-Sekretion bei Menschen mit langjährigem Typ-2-Diabetes verbessert: Es kam zur „Restauration“ der Betazell-Funktion [3]. Eine weitere Beobachtung betraf die Insulinsekretion nach bariatrischer Chirurgie: Sie verbesserte sich drastisch $[4,5]$.

Neue Antidiabetika statt Insulin I Einige Studien haben gezeigt, dass die Gabe von GLP1-RezeptorAgonisten anstelle von Basal-Insulin oder sogar auch Mahlzeiten-Insulin

- eine äquipotente Einstellung des Glukosestoffwechsels mit weniger Hypoglykämien erlaubt und

- zudem auch noch das Körpergewicht und den Blutdruck positiv verändert [6-12].

Es wird daher mit Recht gefragt, ob Insulin wirklich die effektivste Injektionstherapie bei Diabetes mellitus Typ 2 ist [13]. In dem ADA/EASD-Positionspapier werden z. B. die GLP1-Agonisten als Alternative zur Insulintherapie genannt [11].

Kombinationstherapie I Durch die Kombination von langwirksamem Basal-Insulin und GLP1-Rezeptor-Agonisten kommt es zu eindrucksvollen
Verbesserungen des Stoffwechsels, aber auch anderer Faktoren des Metabolischen Syndroms - ein weiterer Hinweis dafür, daß die Gabe von Mahlzeiteninsulin nicht unbedingt immer nötig ist. Die Kombination neuerer oraler Ansätze kann den Insulin-Bedarf verschieben: So verbessert eine Dreifach-Kombination oraler Antidiabetika, z. B.

- Metformin + DPP4-Hemmer + SGLT2-Hemmer u.a. über insulinunabhängige Mechanismen den Stoffwechsel [14]. Dies kann die Notwendigkeit einer Insulintherapie verzögern.

Verschiedene Insulinformen | Es muss auch die Frage beantwortet werden, was mit Insulintherapie genau gemeint ist und wie die Datenlage aussieht, z. B. bei folgenden Varianten:

- Basal-Insulin: Die orale Therapie muss weitergeführt werden, ein einfacher Einstieg ist möglich, die Prognose verbessert sich jedoch nicht.

- Supplementäre Insulintherapie: Die Gabe von kurzwirksamem Insulin soll postprandiale Spitzen therapieren.

- Basal-Bolus-Therapie (langwirksames Basalund kurzwirksames Mahlzeiten-Insulin): Die orale Therapie kann (teilweise) weitergeführt werden oder wird komplett abgesetzt.

Bis heute fehlen saubere randomisierte kontrollierte Studien, die die Überlegenheit einer der Insulin-Varianten gegenüber der konvenitonellen Therapie zeigen.

Mehr Daten! I Die derzeitige Datenlage für die (frühe) Insulintherapie ist dünn. Sinnvollerweise sollte diese am besten gleich gegen die neuen Nicht-Insulin-Therapeutika getestet werden. Denn diese haben wenigstens über einen Zeitraum von 3-4 Jahren gezeigt, dass sie im Vergleich zum Insulin bei gleichwertiger Glukosesenkung

- weniger Hypoglykämien,

- weniger Gewichtszunahme und

- einen geringeren Blutdruck

mit sich bringen. Dies ist aus der Sicht des modernen kardio-metabolischen Risikomanagements bei Typ-2-Diabetes vorteilhaft. Außerdem gibt es für „die Neuen“ auch Daten zur kardiovaskulären Sicherheit (EXAMINE, SAVOR, TECOS, ELIXA [15-18] und sogar Überlegenheit [19, 20]. Leider ist die Bedeutung dieser neuen Erkenntnisse (noch) nicht überall erkannt, so dass diese Optionen noch nicht routinemäßig in die Therapieentscheidung einbezogen werden.

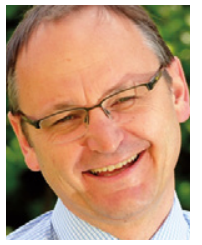

Prof. Dr. med. Stephan Jacob ist Internist, Endokrinologe, Diabetologe, Hypertensiologe, Ernährungsmediziner und Kardio-vaskulärer Präventionsmediziner DGPR. Er arbeitet in der Praxis für Prävention und Therapie Villingen-Schwenningen Prof.Dr.Jacob@web.de

Vollständiges Literaturverzeichnis unter http://dx.doi. org/10.1055/s-0042-102890

Interessenkonflikt Der Autor gibt an, dass er Vortrags- / Beraterhonorare, Reisekostenzuschüsse oder Forschungsförderung von folgenden Firmen erhalten hat: Abbott, AstraZeneca, Bayer, Berlin Chemie, Boehringer Ingelheim, Daichi-Sankyo, Janssen, Johnson \& Johnson, Lilly, Merck, MSD, Novo Nordisk, Novartis, Orexigen, Roche, Sanofi Aventis, Servier.

DOI 10.1055/s-0042-102890

Dtsch Med Wochenschr 2016; 141: 579

(c) Georg Thieme Verlag KG . Stuttgart · New York . ISSN 0012-0472 


\section{Literatur}

1 Holman RR. Assessing the potential for alphaglucosidase inhibitors in prediabetic states. Diabetes Res Clin Pract 1998; 40 (Suppl.): S21-25

2 ORIGIN Trial Investigators, Gerstein HC, Bosch ], Dagenais GR et al. Basal insulin and cardiovascular and other outcomes in dysglycemia. N Engl ] Med 2012; 367: 319-328

3 Jakubowicz D, Wainstein,, Bo Ahrén B et al. High-energy breakfast with low-energy dinner decreases overall daily hyperglycaemia in type 2 diabetic patients: a randomised clinical trial Diabetologia 2015; 58: 912-919

4 Panunzi I, Carlsson L, De Gaetano A et al. Determinants of diabetes remission and glycemic control after bariatric surgery. Diabetes Care 2016; 39: 166-174

5 Steven S, Hollingsworth KG, Small PK et al. Weight loss decreases excess pancreatic triacylglycerol specifically in type 2 diabetes. Diabetes Care 2016; 39: 158-165

6 Russell-Jones D, Vaag A, Schmitz O et al.; Liraglutide Effect and Action in Diabetes 5 (LEAD-5) met+SU Study Group. Liraglutide vs insulin glargine and placebo in combination with metformin and sulfonylurea therapy in type 2 diabetes mellitus (LEAD-5 met+SU): a randomised controlled trial. Diabetologia 2009; 52: 2046-2055

7 Giorgino F, Benroubi M, Sun JH et al. Efficacy and safety of once-weekly dulaglutide versus insulin glargine in patients with type 2 diabetes on metformin and glimepiride (AWARD-2). Diabetes Care 2015; 38: 2241-2249

8 Weissman PN, Carr MC, Ye U et al. HARMONY 4: randomised clinical trial comparing once-weekly albiglutide and insulin glargine in patients with type 2 diabetes inadequately controlled with metformin with or without sulfonylurea. Diabetologia 2014; 57: 2475-2484

9 Diamant M, Van Gaal L, Guerci B et al. Exenatide once weekly versus insulin glargine for type 2 diabetes (DURATION-3): 3-year results of an open-label randomised trial. Lancet Diabetes Endocrinol 2014; 2: 464-473

10 Diamant M, Nauck MA, Shaginian R et al.; 4B Study Group. Glucagon-like peptide 1 receptor agonist or bolus insulin with optimized basal insulin in type 2 diabetes. Diabetes Care 2014; 37: 2763-2773
11 Inzucchi SE, Bergenstal RM, Buse JB et al. Management of hyperglycaemia in type 2 diabetes, 2015: a patient-centred approach. Update to a position statement of the American Diabetes Association and the European Association for the Study of Diabetes. Diabetologia 2015; 58: 429-442

12 Freemantle N, Mamdani M, Vilsbøll T et al. IDegLira versus alternative intensification strategies in patients with type 2 diabetes inadequately controlled on basal insulin therapy. Diabetes Ther 2015; 6: 573-591

13 Buse JB, Peters A, Russell-Jones D et al. Is insulin the most effective injectable antihyperglycaemic therapy? Diabetes Obes Metab 2015; 17: 145-151

14 Matthaei S, Bowering K, Rohwedder K et al.; Study 05 Group. Durability and tolerability of dapagliflozin over 52 weeks as add-on to metformin and sulphonylurea in type 2 diabetes. Diabetes Obes Metab 2015; 17: 1075-1084

15 Scirica BM, Bhatt DL, Braunwald E et al. Saxagliptin and cardiovascular outcomes in patients with type 2 diabetes mellitus. N Engl J Med 2013; 369: 1317-1326

16 Green JB, Bethel MA, Armstrong PW et al; TECOS Study Group: Effect of sitagliptin on cardiovascular outcomes in type 2 diabetes. N Engl ] Med 2015; 373: 232-242

17 White WB, Cannon CP, Heller SR et al.; EXAMINE Investigators. Alogliptin after acute coronary syndrome in patients with type 2 diabetes. $N$ Engl J Med 2013; 369: 1327-1335

18 Pfeffer MA, Claggett B, Diaz R et al.; ELIXA Investigators. Lixisenatide in patients with type 2 diabetes and acute coronary syndrome. N Engl ] Med 2015; 373: 2247-2257

19 Zinman B, Wanner C, Lachin JM et al.; EMPA-REG OUTCOME Investigators. Empagliflozin, cardiovascular outcomes, and mortality in type 2 diabetes. $\mathrm{N}$ Engl J Med 2015; 373: 2117-2128

20 PR Newswire. Victoza ${ }^{\circledR}$ significantly reduced the risk of major adverse cardiovascular events in the LEADER Trial. http://www.prnewswire.com/ news-releases/victoza-significantly-reduced-therisk-of-major-adverse-cardiovascular-events-inthe-leader-trial-300231181.html 